\title{
Le jeu des chiffres
}

\section{Yvonne Gilli}

Dr méd., membre du Comité central de la FMH, responsable du département Numérisation/eHealth

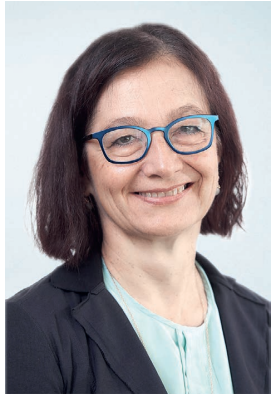

Jouer avec les chiffres est à la mode en ce moment et, dans tous les jeux, il y a des gagnants et des perdants. Comme par hasard, dans le secteur de la santé, les gagnants sont souvent ceux qui définissent les règles du jeu et les perdants, certains groupes de médecins. Fin octobre dernier, l'Office fédéral de la santé publique (OFSP) a publié son jeu des chiffres sur le revenu des médecins [1]. Après une longue période de calcul savant, l'Office fédéral a réussi à démontrer que la moitié des spécialistes installés en cabinet avaient un revenu annuel supérieur à 257000 francs. Les vrais "profiteurs» étaient enfin identifiés et mis sur la place publique, à savoir les neurochirurgiens et les gastroentérologues. Les médecins salariés ont eu droit à une appréciation légèrement plus positive, même s'ils ne sont pas non plus à plaindre avec, pour la moitié la mieux lotie, un revenu supérieur à 197000 francs.

Au début de l'automne, Lukas Brunner, responsable des contrôles d'économicité de tarifsuisse, a aussi présenté son jeu des chiffres, cette fois sur les coûts de la médecine complémentaire à la charge de l'assurance-maladie obligatoire [2]. Grâce à une analyse statistique habile, il a également réussi à démontrer que la médecine complémentaire engendrait des coûts nettement plus élevés que la médecine conventionnelle. Ce n'est que peu avant Noël que le tollé a été provoqué lorsque les chiffres présentés pendant l'événement clients de tarifsuisse ont été communiqués aux médias.

\section{En automne, tarifsuisse a présenté les coûts de la médecine complémentaire à la charge de l'assurance-maladie obligatoire.}

Ces deux jeux de nombres partagent quelques similitudes. Tous deux avancent des résultats qui contredisent des analyses existantes, basées sur des sources de données fiables, sans pour autant les aborder de manière différenciée. Alors que l'étude commandée par l'OFSP souligne seulement que les analyses antérieures ne tenaient pas compte de l'augmentation du travail à temps partiel et n'étaient pas suffisamment représentatives, celle de tarifsuisse ne mentionne pas un mot des résultats précédents. Il est intéressant de rappeler que, dans sa communication, l'OFSP contredit courageusement les résultats d'un relevé de données plus récent de l'Office fédéral de la statistique. Pour sa part, Monsieur Brunner de tarifsuisse ignore à la fois les résultats un peu plus anciens du programme d'évaluation de la médecine complémentaire [3] et une étude hollandaise récente [4].

\section{Les deux études contredisent des analyses} basées sur des sources de données fiables sans les aborder de manière différenciée.

Pourtant, ni l'OFSP ni tarifsuisse n'ont menti. Les indicateurs choisis pour les deux études ont débouché sur leurs résultats respectifs après une évaluation statistique usuelle. Les deux études présentent leurs résultats comme une affirmation, sans questionnement ni remise en question. On peut se demander si une quelconque intention ne vient pas sous-tendre ces analyses. Le conseiller fédéral A. Berset ne chercherait-il pas à légitimer sa prochaine intervention tarifaire en vue de déprécier l'indemnisation des prestations médicales? Les responsables des assureurs-maladie ne chercheraient-ils pas à légitimer le modèle d'affaires intégrant les prestations de médecine complémentaire dans les assurances complémentaires? Imposer une direction et dresser les différents groupes de médecins entre eux - divide et impera: il est bien certain que ce jeu n'a pas que des mauvais effets pour tout le monde.

\section{Références}

1 https://www.bag.admin.ch/bag/fr/home/das-bag/aktuell/news/ news-29-10-2018.html

2 Komplementärmedizin in der OKP: Eine statistische Analyse der Kosten, Kundenevent von tarifsuisse, 18 septembre 2018, Bern. Lukas Brunner, Leiter Wirtschaftlichkeitsprüfungen, und Boris Kaiser, B.S.S. Volkswirtschaftliche Beratung.

3 Programme d'évaluation de la médecine complémentaire (PEK). D. Melchart, F. Mitscherlich, M. Amiet, R. Eichenberger, P. Koch, rapport final, 2005.

4 BMJ open: A 6-year comparative economic evaluation of healthcare costs and mortality rates of Dutch patients from conventional and CAM GPs. Baars EW, et al. BMJ Open 2014;4:e005332. doi:10.1136/bmjopen-2014-005332. 